\title{
Publisher Correction: Cardiovascular Outcome Trials of Diabetes and Obesity Drugs: Implications for Conditional Approval and Early Phase Clinical Development
}

\author{
Andrew J. Krentz ${ }^{1}$ Gerardo Rodriguez-Araujo ${ }^{1}$
}

Published online: 31 January 2018

(C) Springer International Publishing AG, part of Springer Nature 2018

\section{Publisher Correction: Pharm Med \\ https://doi.org/10.1007/s \\ 40290-017-0209-3}

The original version of this article unfortunately contained a mistake. Table 1 was presented incorrectly. The corrected Table 1 is given below.

Table 1 MACE primary composite endpoints applied in diabetes CVOTs to date

\begin{tabular}{ll}
\hline Three-point MACE & Four-point MACE \\
\hline CV death & CV death \\
Non-fatal myocardial infarction & Non-fatal myocardial infarction \\
Non-fatal stroke & Non-fatal stroke \\
& Hospitalization for unstable angina
\end{tabular}

MACE major adverse cardiovascular events, $C V$ cardiovascular, CVOTs cardiovascular outcome trials

The original article can be found online at https://doi.org/10.1007/ s40290-017-0209-3.

\footnotetext{
Andrew J. Krentz

andrew.krentz@prosciento.com

1 ProSciento, 855 3rd Avenue Suite 4400, Chula Vista, CA

91911, USA
} 